\title{
Fredholm Determinants and the $\tau$ Function for the Kadomtsev-Petviashvili Hierarchy
}

By

\author{
Ch. PöPPE* and D. H. SATTINGER**1
}

\begin{abstract}
The "dressing method" of Zakharov and Shabat is applied to the theory of the $\tau$ function, vertex operators, and the bilinear identity obtained by Sato and his co-workers. The vertex operator identity relating the $\tau$ function to the Baker-Akhiezer function is obtained from their representations in terms of the Fredholm determinants and minors of the scattering operator appearing in the Gel'fand-Levitan-Marchenko equation. The bilinear identity is extended to wave functions analytic in a left half plane and is proved as a consequence of the inversion theorem and the convolution theorem for the Laplace transform.
\end{abstract}

\section{Contents}

1. Introduction

2. The dressing method of Zakharov-Shabat

3. Fredholm determinants and minors

4. The bilinear identity

5. The vertex operator

6. Positivity of the $\tau$ function; special solutions

\section{§ 1. Introduction}

One way to characterize the class of "soliton equations" is through the existence of a linearizing transformation, i.e. a transformation that maps the nonlinear soliton equation into a corresponding linear equation. Originally, this transformation was accomplished via the properties of the eigenfunctions ("wave functions") of an auxiliary "isospectral"

Communicated by H. Araki, August 24, 1987

* Universität Heidelberg, Sonderforschungsbereich 123, Im Neuenheimer Feld 294, D-6900 Heidelberg, Germany

** School of Mathematics, University of Minnesota, Minneapolis, Minnesota, 55455, U. S. A. Research supported in part by NSF Grant DMS-87-02758. 
problem: the "inverse scattering transform". Zakharov and Shabat's "dressing method" $[19,23,24]$ is a reformulation of the inverse scattering transform that avoids explicit use of the wave functions and deals instead with their (suitably defined) Fourier transforms.

For example, the Korteweg deVries (KdV)equation

$$
u_{t}=\frac{1}{4}\left(u_{x x x}+6 u u_{x}\right)
$$

has as isospectral operator the Schrödinger operator $L=D^{2}+u$, where $D=\partial / \partial x$. The operator $L$ is related to the "bare" operator $L_{0}=D^{2}$ by the "dressing equations"

$$
L\left(1+K_{ \pm}\right)=\left(1+K_{ \pm}\right) L_{0}
$$

where the operators $K_{ \pm}$are Volterra integral operators:

$$
K_{+} \phi(x)=\int_{x}^{\infty} K_{+}(x, z) \phi(z) d z, \quad K_{-} \phi(x)=\int_{-\infty}^{x} K_{-}(x, z) \phi(z) d z
$$

The wave functions of $L$ are obtained from those of $L_{0}$, namely $e^{i k x}$, by applying the dressing operators $\left(1+K_{ \pm}\right)$. Thus, for example,

$$
\Psi_{+}(x)=\left(1+K_{+}\right) e^{i k x}=e^{i k x}+\int_{x}^{\infty} K_{+}(x, z) e^{i k z} d z
$$

satisfies $\left(L+K^{2}\right) \Psi=0$.

The dressing method focuses on the kernels $K_{ \pm}$as the primary object rather than the wave functions. An easy calculation (see §2) shows that if $K_{ \pm}$both dress $L_{0}$ to $L$, then the integral operator $F$ defined by

$$
(1+F)=\left(1+K_{+}\right)^{-1}\left(1+K_{-}\right)
$$

commutes with $L_{0}$, i. e solves a linear equation. The linearizing transformation $L \rightarrow F$ will be referred to as the "dressing transformation." The inverse dressing transformation involves solving (1.1) for $K_{ \pm}$given $F$. It is then easy to recover $L$ from $K_{+}$or $K_{-}$(the details will be given in $\S 2$ ).

Equation (1.1) is equivalent to the Gel'fand-Levitan-Marchenko (GLM) equation of inverse scattering theory. It can be solved by considering it as a family of Fredholm integral equations of the for $m$

$$
F_{(x)}+K_{+(x)}+K_{+(x)} F_{(x)}=0
$$

where $F_{(x)}$ is a "truncation" of $F$ (cf. $\S 3$ ).

The technique of the dressing method was formally extended to 
the Kadomtsev-Petviashvili (KP) equation, for which the appropriate isospectral operator is a partial differential operator in two variables, in [23]. In fact, the entire KP hierarchy of commuting flows can be obtained by dressing the operators $\partial / \partial x_{n}-D^{n}$. Specifically, consider the differential operators $\partial / \partial x_{n}-B_{n}$ obtained via the dressing

$$
\left(\frac{\partial}{\partial x_{n}}-B_{n}\right)\left(1+K_{ \pm}\right)=\left(1+K_{ \pm}\right)\left(\frac{\partial}{\partial x_{n}}-D^{n}\right) \text {. }
$$

Each operator $B_{n}$ is a differential operator of order $n$ beginning with $D^{n}$. The coefficients of the $B_{n}$ are assumed to depend on infinitely many variables $x_{1}, x_{2}, \ldots$, though each individual coefficient involves only a finite number of them. The KP hierarchy is obtained from the commutation relations

$$
\left[\frac{\partial}{\partial x_{n}}-B_{n}, \frac{\partial}{\partial x_{m}}-B_{m}\right]=0
$$

The KP equation itself is obtained from (1.3) for $n=2$ and $m=3$. It is

$$
\left(u_{t}-\frac{1}{4}\left(u_{x x x}+6 u u_{x}\right)\right)_{x}=\frac{3}{4} u_{y y}
$$

where $x=x_{1}, y=x_{2}, t=x_{3}, \ldots$

In a completely independent approach, originating in holonomic quantum field theory, Sato [17], and Date, Jimbo, Kashiwara, and Miwa [4], developed a formalism for the KP hierarchy which introduced fundamentally new ideas into the subject of integrable systems.

Of the many important results of their theory, we focus on the following in the present paper.

(i) the " $\tau$ function": each of the solutions of the KP hierarchy (i.e. every coefficient of the $B_{n}$ ) can be expressed as some derivative of the logarithm of a single function, the $\tau$ function, of all the variables $x_{1}, x_{2}, \ldots$;

(ii) the "bilinear identity": a contour integral identity involving the wave function of the KP hierarchy and its adjoint wave function; it can be turned into an identity for the $\tau$ function which gives a generating function for an infinite hierarchy of bilinear differential equations, the Hirota equations. This establishes the link with Hirota's [6] bilinear formalism for soliton equations, since the variable in which his bilinear differential equations are formulated can be identified 
with the $\tau$ function.

(iii) the "vertex operator": an algebraic operation that allows one to recover the wave function as a quotient of the $\tau$ function and some "translate" of it, thereby bypassing the need of explicitly solving the GLM equation.

The $\tau$ function thus, in some sense, carries all the information about the solution of the hierarchy.

In this paper we attempt to synthesize the ideas of Zakharov and Shabat and the "Kyoto school." In [4] the theory is developed using the algebra of formal pseudo-differential operators

$$
P(x, D)=\sum_{j=-\infty}^{N} P_{,}(x) D^{\prime}
$$

as the dressing transformations. These pseudo-differential operators are naturally interpreted as symbols for the Volterra integral operators of the dressing method.

We describe in $\S 2$ the Zakharov-Shabat dressing method as it applies to the KP hierarchy and give its connections to the work of the Kyoto group.

In $\S 3$ we prove the important fact (observed briefly in [7]) that the $\tau$ function is identical to the Fredholm determinant of the truncated operator $F_{(x)}$ occuring in the GLM equation (1.1') using results from classical Fredholm determinant theory combined with the dressing method. The Fredholm determinant method has previously been applied to the $\mathrm{KdV}$ equation by Oishi [10], and to the sine-Gordon and $\mathrm{KdV}$ equations by Pöppe $[12,13]$.

In $\S 4$ we give a proof of the bilinear identity based on the Volterra integral operator representation of the dressing transformations. Instead of using Laurent expansions of the wave functions, convergent outside some suitably large disk, as in [4], we use the Laplace inversion theorem and the convolution theorem. This simplifies the proof somewhat and extends the validity of the bilinear identity to wave functions analytic in some half plane.

In $\S 5$ we obtain the vertex operator relation directly from the representation of the $\tau$ function and the wave function in terms of Fredholm determinants and minors of the GLM equation. The proof is related to some ideas of Rosales for the $\mathrm{KdV}$ equation [15].

Since all the functions in the KP hierarchy are logarithmic deri- 
vatives of $\tau$, it must be positive in order for the solutions to be regular. In $\S 6$ we give sufficient conditions for this positivity, as well as some counterexamples.

The Fredholm determinant method gives a concrete representation of the $\tau$ function for the initial value problem for the KP(I) hierarchy. It also raises fundamental questions about the extent of the dressing method in the context of multi-dimensional isospectral problems. Inverse problems connected with multi-dimensional isospectral operators can lead to $\bar{\partial}$ problems in which the wave function $w(x, k)$ may not be analytic anywhere in the complex plane (cf. [2], [3], [25]). This happens in the case of the KP hierarchy for real values of the variables $x_{2 j}$. This case has been called "KP II" by Ablowitz, Bar Yaacov, and Fokas, or the "stable" case by Zakharov and Shabat. The treatment of the general initial value problem for this case requires the use of the $\tilde{\partial}$ method. The dressing method, if it applies at all to this case, will have to be substantially modified. When the $x_{2 j}$ are imaginary (the "KP I", or "unstable," case), the wave function $w$ is analytic in some half $\mathrm{k}$-plane, and the associated isospectral problem leads to a non-local Riemann-Hilbert problem which is equivalent to $\left(1.1^{\prime}\right)$. The solution of the initial value problem for KP I by the GLM equation has been discussed by Manakov [8].

The $\tau$ function for the multi-soliton solutions of KP I can be analytically continued to real $x_{2 j}$ to obtain multi-soliton solutions for KP II. In general, however, additional constraints must be placed on the parameters to ensure that the analytic continuation is real and positive. This is illustrated by some of the examples in $\S 6$.

A theory of the $\tau$ function from the Riemann-Hilbert point of view has been developed by Segal and Wilson [18, 22]. In their approach the $\tau$ function is obtained as a determinant of a certain projection operator on a Hilbert space. They consider the Grassmanian of closed subspaces $W$ of the Hilbert space $H=L_{2}\left(S^{1}\right), S^{1}$ being the unit circle. $H$ is decomposed into the direct sum of subspaces $H_{ \pm}$ spanned by $\left\{z^{k}\right\}$ for $k \geq 0$ and $k<0$ respectively. Subspaces $W$ are considered for which the projection $W \rightarrow H_{+}$is a Fredholm operator of index zero. The $\tau$ function is given in terms of this projection. 
It would be desirable to develop a theory of the $\tau$ function that is less dependent on the specific properties of Fredholm determinants, but rather focuses on more general properties of determinants. For example, a $\tau$ function for the rational solutions of the KP equation can be obtained as a determinant of a finite dimensional matrix which does not come from the GLM equation. These matters are presented in a separate paper by Pöppe [14].

The authors would like to acknowledge helpful discussions with Professors M.J. Ablowitz and A.S. Fokas concerning $\bar{\partial}$ methods, the theory of the KP II equation, and the relationship of $\bar{\partial}$ methods to the GLM approach to inverse scattering.

\section{§ 2. The Dressing Method of Zakharov-Shabat}

Date et al [4] used the calculus of pseudo-differential operators introduced by Gel'fand and Dikii [5]. The method of differential algebra has proved a powerful and elegant tool in the subject of integrable systems (cf. Wilson, [21], for example). The operator $D=d / d x$ is formally represented by a symbol $\partial$, and an algebraic formalism is developed for pseudo-differential operators

$$
P(x, \partial)=\sum_{j=-\infty}^{N} P_{j}(x) \partial^{j}
$$

However, these formal algebraic manipulations omit some essential analytical features which are fundamental to the theory. Properly speaking, the expression $P(x, \partial)$ above is the symbol for an integro differential operator. The operator itself is realized formally from the transformation pair

$$
P(x, \partial) u(x)=\int_{-\infty}^{\infty} e^{i s x} P(x, s) U(s) d s \quad U(s)=\frac{1}{2 \pi} \int_{-\infty}^{\infty} e^{-i s x} u(x) d x
$$

except that $P(x, \partial)$ has an essential singularity at the origin. The Fourier inversion formula may be interpreted by indenting the contour above or below the origin; and in that case one obtains upper or lower Volterra integral operators with $P(x, \partial)$ as their symbol. This amounts to interpreting $\partial^{-1}$ either as

$$
\partial^{-1} \phi(x)=\int_{-\infty}^{x} \phi(s) d s \quad \text { or as } \quad \partial^{-1} \phi(x)=-\int_{x}^{\infty} \phi(s) d s .
$$


In the dressing method this difference is brought to the fore. In this approach one deals with the Volterra integral operators themselves instead of their symbols :

$$
K_{+} \Psi(x)=\int_{x}^{\infty} K_{+}(x, y) \psi(y) d y \quad \text { and } \quad K_{-} \Psi(x)=\int_{-\infty}^{x} K_{-}(x, y) \Psi(y) d y .
$$

Suppose we consider such operators and ask that $L\left(1+K_{ \pm}\right)=\left(1+K_{ \pm}\right) D^{2}$, where $L$ is the Schrödinger operator $L=D^{2}+u$. We say that $K_{ \pm}$dress $D^{2}$ to the operator $\mathrm{L}$. It is an easy exercise to see that the kernels $K_{ \pm}$must satisfy the characteristic boundary problem

$$
\begin{aligned}
& u(x)-2 \frac{d}{d x} K(x, x)=0 \\
& K_{x x}(x, z)-K_{z z}(x, z)+u(x) K(x, z)=0 .
\end{aligned}
$$

Furthermore, if $K_{+}(x, z)$ decays as $z \rightarrow \infty$, then $\Psi_{+}=\left(1+K_{+}\right) e^{i k x}$ is well defined for $\operatorname{Im} \mathrm{k}>0$, satisfies $\left(L+k^{2}\right) \Psi_{+}=0$, and $\Psi_{+} \sim e^{i k x}$ as $x \rightarrow$ $+\infty \Psi_{+}$is the wave function for the isospectral operator $L ; \Psi_{+}-e^{i k x}$ is the Fourier transform of the dressing kernel $K_{+}$with respect to the second variable.

Given a "bare" differential operator $L_{0}$ (for example, $L_{0}=d^{2} / d x^{2}$ ) Zakharov and Shabat ask "Under what conditions is $L$, defined by $L\left(1+K_{+}\right)=\left(1+K_{+}\right) L_{0}$, a pure differential operator?" In general, $L$ will consist of a differential part and a Volterra part. They answer the question in the following way. Suppose we also dress $L_{0}$ from $-\infty$, i. e. we construct $L^{\prime}$ by $L^{\prime}\left(1+K_{-}\right)=\left(1+K_{-}\right) L_{0}$ for some lower Volterra operator $K_{-}$. Under what conditions do we get the same operator? To answer this question, Zakharov and Shabat introduce the integral operator $(1+F)$ given by (1.1) or, equivalently

$$
\left(1+K_{+}\right)(1+F)=1+K_{-} .
$$

We shall assume in what follows that $\left(1+K_{ \pm}\right)^{-1}$ are defined on $C_{0}^{\infty}(R)$. This is indeed the case in numerous important specific examples, such as the $N$-soliton case. For the $N$-soliton case, for example, one can find an $F(x, z)$ that decays exponentially as $z \rightarrow \infty$; and then one sees that $K_{+}(x, z) \rightarrow 0$ exponentially as $z \rightarrow \infty$. On the other hand, $K_{-}$grows exponentially as $z \rightarrow-\infty$ (cf. the one-soliton solution below); but if we restrict ourselves to the dense set $C_{0}^{\infty}(R)$, then $\left(1+K_{ \pm}\right)^{-1}$ can be constructed by a Neumann series. 
Theorem 2.1. The dressing of $L_{0}$ from $+\infty$ and $-\infty$ is the same iff $(1+F)$ commutes with $L_{0}$.

Proof: If $K_{ \pm}$both dress $L_{0}$ to $L$ then we have

$$
L\left(1+K_{-}\right)=L\left(1+K_{+}\right)(1+F)=\left(1+K_{+}\right) L_{0}(1+F)
$$

on the one hand, and

$$
L\left(1+K_{-}\right)=\left(1+K_{-}\right) L_{0}=\left(1+K_{+}\right)(1+F) L_{0}
$$

on the other. Assuming $\left(1+K_{+}\right)$is invertible, we find that $\left[L_{0}, F\right]=0$.

Conversely, if $\left[L_{0}, F\right]=0$ then

$$
\begin{aligned}
& \left(1+K_{-}\right) L_{0}\left(1+K_{-}\right)^{-1}=\left(1+K_{+}\right)(1+F) L_{0}\left(1+K_{-}\right)^{-1} \\
& \quad=\left(1+K_{+}\right) L_{0}(1+F)\left(1+K_{-}\right)^{-1}=\left(1+K_{+}\right) L_{0}\left(1+K_{+}\right)^{-1},
\end{aligned}
$$

so that $K_{+}$and $K_{-}$both dress $L_{0}$ to the same operator.

On a group theoretical level this is a natural result. If we consider all the transformations $\left(1+K_{ \pm}\right)$as forming a group, then the result says that two transformations $P_{1}$ and $P_{2}$ in this group intertwine $L_{0}$ and $L$ iff $P_{2}^{-1} P_{1}$ commutes with $i_{0}$. The transformations which commute with $L_{0}$ form a subgroup (say $H\left(L_{0}\right)$ ) of the group of transformations (the isotropy group of $L_{0}$ ). So the result says that all transformations which dress $L_{0}$ to $L$ lie in the same left coset of $H\left(L_{0}\right)$.

There is, however, a caveat to this picture which becomes apparent when one begins to apply the method to specific cases in the $\mathrm{KdV}$ or KP equations. Namely, one discovers that there is an asymmetry between $K_{+}$and $K_{-}$, so that $1+K_{+}$, say, is invertible, while $1+K_{-}$is not. (See the example below for the one-soliton solution.)

Theorem 2.2. If $\left(1+K_{-}\right) L_{0}=L\left(1+K_{-}\right)$and $\left(1+K_{+}\right) L_{0}=L\left(1+K_{+}\right)$, then $L$ is a purely differential operator.

Proof: The dressing of $L_{0}$ by $1+K_{-}$consists of a differential part plus a lower Volterra integral operator; while the dressing of $L_{0}$ by $1+K_{+}$consists of a differential part plus an upper Volterra integral operator. The difference of the two dressings thus consists of a differential part, plus a lower Volterra integral operator plus an upper Volterra integral operator, and the sum of these three 
operators is zero.

We claim that each component must also be zero. For, let $T=$ $P(x, D)+V_{+}+V_{-}$where $P$ is a differential operator, and $V_{+}$and $V_{-}$ are upper and lower Volterra operators. Applying $T$ to a delta function with support at the point $x=a$, we find that

$$
T \delta_{a}= \begin{cases}V_{+}(x, a) & x<a \\ V_{-}(x, a) & x>a\end{cases}
$$

Since $T=0$, this implies that the kernels $V_{ \pm}(x, a)$ both vanish. It then follows that the differential part $P$ also vanishes. (Note: Since $V_{+}$is an upper Volterra integral operator, the kernel $V_{+}(x, y)=0$ for $x>y$; the reverse holds for $V_{-.}$)

Given the Schrödinger operator $L=D^{2}+u$ we can construct the integral operators $K_{ \pm}$in one of two equivalent ways. If we require that $L\left(1+K_{ \pm}\right)=\left(1+K_{ \pm}\right) D^{2}$ then we get a hyperbolic differential equation for the kernels $K_{ \pm}$as in (2.1). Under certain boundary conditions at $\pm \infty$, these have unique solutions. On the other hand, we could take the wave functions $\Psi(x, k)$ of $L$ and try to represent them as $\Psi_{ \pm}=\left(1+K_{ \pm}\right) e^{ \pm i k x}, e^{ \pm 2 k x}$ being the wave functions of $D^{2}$. Thus the kernels $K_{ \pm}$are Fourier-Laplace transforms of the wave functions. The kernel $F$ is then obtained by forming $\left(1+K_{+}\right)^{-1}\left(1+K_{-}\right)$, provided $\left(1+K_{+}\right)^{-1}$ exists. This is a solution of the "forward scattering problem" in the context of the dressing method.

Now consider the inverse problem. Suppose $F$ is known and we want to find the potential $u$. From the first equation in (2.1) we see that it suffices to find the Volterra integral operators $K_{ \pm}$such that $\left(1+K_{+}\right)(1+F)=1+K_{-}$. Writing this out we obtain

$$
K_{+}(x, y)+F(x, y)+\int_{-\infty}^{\infty} K_{+}(x, z) F(z, y) d z=K_{-}(x, y) .
$$

But $K_{+}(x, y)=0$ if $y<x$ and $K_{-}(x, y)=0$ if $y>x$; so this integral equation reduces to

$$
K_{+}(x, y)+F(x, y)+\int_{x}^{\infty} K_{+}(x, z) F(z, y) d z=0 \text { if } y>x,
$$

which is the Gel'fand-Levitan-Marchenko integral equation of inverse scattering theory for the $\mathrm{KdV}$ equation. Once $K_{+}$is found we can easily compute $K_{-}$from the original integral equation by setting $y<x$. Once $K_{ \pm}$are determined we can find the potential $u$ 
from the first equation in (2.1). This constitutes a solution of the inverse scattering problem in the context of the dressing method.

For the case of the $\mathrm{KdV}$ hierarchy, $F(x, z)=F(x+z)$, i. e. the kernel is "additive", and the GLM equation, as is well known, is equivalent to a local Riemann-Hilbert problem. For the KP hierarchy, this is no longer the case, and the GLM equation is equivalent to a non-local Riemann-Hilbert problem ( $[1,8,25])$.

The KP hierarchy is obtained by dressing the family of multidimensional operators $\partial / \partial x_{n}-D^{n}, n=2,3, \ldots$ Let $K_{ \pm}$and $B_{n}$ be operators satisfying the dressing equation (1.2):

$$
\left(\partial / \partial x_{n}-B_{n}\right)\left(1+K_{ \pm}\right)=\left(1+K_{ \pm}\right)\left(\partial / \partial x_{n}-D^{n}\right) .
$$

The operators $B_{n}$ are $n^{\text {th }}$ order differential operators with leading term $D^{n}$. The process of obtaining the differential operators $B_{n}$ is an algebraic one (cf. [23] p. 227). The coefficients of the lower order derivatives are determined by applying the above operator identities to a function $\Psi$ and integrating all the terms in the integrands by parts. When this is done one ends up with integral terms (non-local operators) and local operators on $\Psi$. The $B_{n}$ are determined from the local operations; while the integral terms give differential equations for the kernel $K$. (Here $K$ stands for both $K_{+}$and $K_{-}$)

Note that the differential equations and the boundary conditions on the diagonal $x_{1}=z_{1}$ are identical for both $K_{+}$and $K_{-}$. This is a manifestation of the fact that all kernels which satisfy the dressing relation (1.2) have the same symbol.

We introduce the hierarchy variable $x=\left(x_{1}, x_{2}, \ldots\right)$. Throughout this paper we also make the convention that $z=\left(z_{1}, x_{2}, x_{3}, \ldots\right), y=$ $\left(y_{1}, x_{2}, x_{3}, \ldots\right)$, etc. Though this notation is redundant in an expression like $F(x, z)$ or $K_{+}(x, z)$, it will nevertheless prove useful.

The operators $B_{2}$ and $B_{3}$, for example, are given by

$$
B_{2}=D^{2}+u(x), \quad B_{3}=D^{3}+(3 / 4)(u D+D u)+w
$$

where

$$
u(x)=2 \frac{d}{d x_{1}} K(x, x) \quad w(x)=\left.\frac{3}{2}\left\{\partial_{x_{1}}^{2}-\partial_{z_{1}}^{2}+u(x)\right\} K(x, z)\right|_{x=z}
$$

The entire hierarchy of operators $B_{n}$ could in principle be determined, though the computations get much more complicated as one 
goes higher.

From these equations for $u$ and $w$, we see that the coefficients of the operators $B_{2}$ and $B_{3}$ are obtained in terms of the dressing kernel $K(x, z)$ and its derivatives with respect to $x_{1}$ and $z_{1}$ on the diagonal $x_{1}=z_{1}$.

For example, the dressing equation for $n=2$, (with $B_{2}=D^{2}+u$ ) works out to

$$
\begin{gathered}
\left\{u(x)-2 \frac{\partial}{\partial x_{1}} K(x, x)\right\} \Psi(x)+\int_{x_{1}}^{\infty}\left\{\left(\partial_{x_{1}}^{2}-\partial_{z_{1}}^{2}-\partial_{x_{2}}\right.\right. \\
+u(x)) K(x, z)\} \Psi(z) d z_{1}=0 .
\end{gathered}
$$

This equation must hold for all $\Psi$, so we must have

$$
\begin{aligned}
& u(x)=2 \frac{\partial}{\partial x_{1}} K(x, x) \\
& \frac{\partial K(x, z)}{\partial x_{2}}-\frac{\partial^{2} K(x, z)}{\partial x_{1}^{2}}+\frac{\partial^{2} K(x, z)}{\partial z_{1}^{2}}-u(x) K(x, z)=0 .
\end{aligned}
$$

The equations of the KP hierarchy are obtained as the integrability conditions (1.3). These commutation relations follow immediately from the commutativity of the family of "bare" operators $\partial / \partial x_{n}-D^{n}$ and the dressing relation (1.2). The KP equation itself arises from (1.3) for $n=2$ and $m=3$ :

$$
\left[\frac{\partial}{\partial y}-D^{2}-u, \frac{\partial}{\partial t}-D^{3}-\frac{3}{4}(u D+D u)-w\right]=0 .
$$

In fact, working out this commutator, we get

$$
\begin{aligned}
& {\left[\frac{\partial}{\partial y}-D^{2}-u, \frac{\partial}{\partial t}-D^{3}-\frac{3}{4}(u D+D u)-w\right]} \\
& \quad=\left(u_{t}-\frac{1}{4}\left(u_{x x x}+6 u u_{x}\right)-w_{y}+w_{x x}-\frac{3}{4} u_{x y}\right)+\left(2 w_{x}-\frac{3}{2} u_{y}\right) D .
\end{aligned}
$$

Setting both terms equal to zero we get $w_{x}=3 / 4 u_{y}$ and

$$
u_{t}-\frac{1}{4}\left(u_{x x x}+6 u u_{x}\right)=w_{y} .
$$

The Kadomtsev-Petviashvili equation follows by differentiating this equation with respect to $x$ :

$$
\left(u_{t}-\frac{1}{4}\left(u_{x x x}+6 u u_{x}\right)\right)_{x}=\frac{3}{4} u_{y y} .
$$

The KP equation was derived by this method by Zakharov and 
Shabat. Their procedure may be extended to derive all the equations in the KP hierarchy by working out the commutators for the operators $B_{n}$.

The kernels $K(x, z)$ formally satisfy the infinite set of differential equations :

$$
\frac{\partial K(x, z)}{\partial x_{n}}-B_{n} K(x, z)+(-1)^{n} D_{z}^{n} K(x, z)=0 .
$$

Here $B_{n}$ acts on $K$ with respect to $x_{1}$ and $D_{z}$ acts on $K$ with respect to $z_{3}$.

The KdV hierarchy is obtained as a special case of the KP hierarchy by dressing the operators

$$
\partial / \partial t_{n}-D^{n} \quad n=1,3,5, \ldots
$$

The even order operators $D^{2 n}$ all dress to pure differential operators in $x_{1}$ for the KdV hierarchy, but not in the KP hierarchy. In particular, $\left(D^{2}+u\right)\left(1+K_{+}\right)=\left(1+K_{+}\right) D^{2}$.

The GLM equation for the KP hierarchy is

$$
K_{+}(x, z)+F(x, z)+\int_{x_{1}}^{\infty} K_{+}(x, y) F(y, z) d y_{1}=0 \quad \text { if } x_{1}<z_{1} \text { 。 }
$$

Let us assume, for the time being, that the integral operator $F$ defined by $1+F=\left(1+K_{+}\right)^{-1}\left(1+K_{-}\right)$is well defined on some dense set of functions. By the argument used in the proof of Theorem 2.2, we find that $B_{n}$ is a purely differential operator iff

$$
\left[F, \partial / \partial x_{n}-D^{n}\right]=0 .
$$

These commutation relations for the integral operator $F$ give partial differential equations for its kernel $F(x, z)$, namely:

$$
\frac{\partial F}{\partial x_{n}}-\frac{\partial^{n}}{\partial x_{1}^{n}} F+(-1)^{n} \frac{\partial^{n}}{\partial z_{1}^{n}} F=0, \quad n=2,3, \ldots
$$

A special solution of this system of linear equations is given by

$$
F(x, z)=e^{\xi(x, p)-\xi(z, q)}
$$

where

$$
\xi(x, k)=\sum_{j=1}^{\infty} x_{j} k^{j} .
$$

We shall see below that expression (2.5) is precisely the form of $F$ that gives rise to a one-soliton solution of the KP hierarchy. Note 
that $F$ decays exponentially as $z_{1} \rightarrow \infty$ if $R e p<0<R e q$.

The function $\xi(x, k)$ plays a prominent role in the treatment by the "Kyoto school." The preceeding discussion shows how naturally this function arises in the Zakharov-Shabat picture.

The wave function $e^{\xi(x, k)}$ satisfies the infinite set of equations

$$
\left(\partial / \partial x_{n}-D^{n}\right) e^{\xi(x, k)}=0 .
$$

A wave function for the $\mathrm{KP}$ hierarchy is given by

$$
w(x, k)=\left(1+K_{+}\right) e^{\xi(x, k)}=e^{\xi(x, k)}+\int_{x_{1}}^{\infty} K_{+}(x, z) e^{\xi(z, k)} d z_{1} .
$$

This wave function corresponds to the wave function $\Psi_{+}$for the $\mathrm{KdV}$ equation. It is analytic in $R e k<0$ provided $K_{+}$remains bounded as $z_{1} \rightarrow \infty$.

Sato and Date et al obtained the KP hierarchy by dressing the operators $D^{n}$ with a pseudo-differential operator $P$. The differential operators $B_{n}$ are obtained as the differential part of the pseudo-differential operator $L^{n}$, where $L=P D P^{-1}$, and $P$ is a pseudodifferential operator. In fact, $P$ is precisely the symbol of either of the Volterra integral operators $\left(1+K_{+}\right)$or $\left(1+K_{-}\right) . L$ plays the role of the isospectral operator in $[4,17]$; while in $[1,2,3,8,23,24,25]$ the isospectral operator is the multidimensional operator $\partial / \partial y-D^{2}-u$.

The elementary solution (2.5) gives the one-solition solution of the KP hierarchy. In order to obtain more general solutions we may form a superposition of such fundamental solutions. We may take

$$
F(x, z)=\iint_{c^{2}} e^{\xi(x, p)-\xi(z, q)} d \mu(p, q)
$$

where $\mu$ is a measure in $C^{2}$, the Cartesian product of the complex plane with itself. For simplicity of notation we shall abbreviate this double integral as

$$
F(x, z)=\int e^{\xi(x, p(s))-\xi(z, q(s))} d \mu(s)
$$

where $s=\left(s_{1}, s_{2}\right)$ and $d \mu(s)=d \mu\left(s_{1}, s_{2}\right)$ is a measure which contains possible delta function terms. In fact, one could even extend this representation and allow $\mu$ to be a distribution containing derivatives of $\delta$ functions. In particular, (2.6) yields as a special case an 
$F$ which is comprised of sums of discrete terms (the solitons) and a term corresponding to the "reflection coefficient."

For example, the choice

$$
F=\sum_{j=1}^{N} a_{j} e^{\xi\left(x, p_{j}\right)-\xi\left(z, q_{j}\right)}
$$

gives rise to the $N$-soliton solution of the KP hierarchy.

The kernels $K_{ \pm}$for the one-soliton solution of the KP hierarchy are easily obtained. We take $F$ as given in (2.5) and obtain $K_{+}$ by solving the GLM equation. In the present case it is a simple matter of carrying out an integration. We find

$$
\begin{aligned}
& K_{+}(x, z)=-\frac{F(x, z)}{\Delta(x)} \quad x_{1}<z_{1} \\
& K_{-}(x, z)=\frac{F(x, z)}{\Delta(x)} \quad \text { for } \quad x_{1}>z_{1}
\end{aligned}
$$

where $F$ is as given in (2.5) and

$$
\Delta(x)=1+\int_{x_{1}}^{\infty} e^{\xi(t, p)-\xi(t, q)} d t_{1}=1-\frac{e^{\xi(x, p)-\xi(x, q)}}{p-q} .
$$

We shall see later that $\Delta(x)$ is the $\tau$ function for the one-soliton solution of the KP hierarchy. Since $F$ decays as $x_{1}, z_{1} \rightarrow \infty$, so does $K_{+}$; but $K_{-}$grows exponentially as $z_{1} \rightarrow-\infty$.

The wave function $w(x, k)$ (also called the Baker-Akhiezer function for the hierarchy), obtained from the relation $w=\left(1+K_{+}\right) e^{\xi(x, k)}$ is easily computed for the one-soliton solution:

$$
\begin{aligned}
w(x, k) & =\left[1+\frac{1}{k-q} \frac{F(x, x)}{\Delta(x)}\right] e^{\xi(x, k)} \\
& =\left[1-\frac{1}{k-q} \frac{\partial}{\partial x_{1}} \log \Delta(x)\right] e^{\xi(x, k)} .
\end{aligned}
$$

Thus $w$ has a simple pole at $k=q$ in the right half plane. Similarly, we may construct a second wave function corresponding to $\Psi_{\text {- for }}$ the $\mathrm{KdV}$ case :

$$
\begin{aligned}
w_{-} & =\left(1+K_{-}\right) e^{-\xi(x, k)} \\
& =\left[1-\frac{e^{\xi(x, p)-\xi(x, q)}}{(k+q) \Delta(x)}\right] e^{\xi(x, k)} \\
& =\left[1+\frac{1}{k+q} \frac{\partial}{\partial x_{1}} \log \Delta(x)\right] e^{-\xi(x, k)} .
\end{aligned}
$$


In the bilinear identity, to be proved in $\S 5$ we shall need the adjoint wave function $w^{*}(x, k)$. (Here, as in [4], the asterisk does not denote the complex conjugate.) This is the wave function for the adjoint KP hierarchy, which is obtained by dressing the operators

$$
\frac{\partial}{\partial x_{n}}+(-1)^{n} D^{n}
$$

The kernel $F^{*}$ for the adjoint hierarchy must satisfy the commutation relations

$$
\left[\frac{\partial}{\partial x_{n}}+(-1)^{n} D^{n}, F^{*}\right]=0
$$

and one finds readily that the elementary solution of this set of equations is $F^{*}(x, z)=F(z, x)=e^{\xi(z, p)-\xi(x, q)}$. From $\left(1+K_{+}\right)(1+F)=$ $\left(1+K_{-}\right)$we find that $\left(1+K_{-}^{t}\right)^{-1}\left(1+F^{t}\right)=\left(1+K_{+}^{t}\right)^{-1}$. The kernel corresponding to $K_{-}$for the adjoint hierarchy is therefore $\left(1+K_{+}^{t}\right)^{-1}$, and $w^{*}$, given by

$$
w^{*}=\left(1+K_{+}^{t}\right)^{-1} e^{-\xi(x, k)},
$$

is a wave function for the adjoint hierarchy. (Note that the transpose of an upper Volterra operator is a lower Volterra operator.)

For the one soliton solution the kernel for the integral operator $\left(1+K_{+}^{t}\right)^{-1}$ is easily found. We solve the resolvent equation $(1+G)$ $=\left(1+K_{+}\right)^{-1}$ and then put $\left(1+K_{+}^{t}\right)^{-1}=\left(1+G^{t}\right)$. This resolvent equation is

$$
K_{+}(x, z)+G(x, z)+\int_{x_{1}}^{z_{1}} K_{+}(x, t) G(t, z) d t_{1}=0, \quad x_{1}<z_{1} .
$$

The solution

$$
G(x, z)=\frac{e^{\xi(x, p)-\xi(z, q)}}{\Delta(z)} \quad x_{1}<z_{1}
$$

is found without difficulty; and then the adjoint wave function for the one-soliton solution of the KP hierarchy is

$$
w^{*}=\left(1+K_{+}^{t}\right)^{-1} e^{-\xi(x, k)}=\left[1+\frac{1}{k-p} \frac{\partial}{\partial x_{1}} \log \Delta(x)\right] e^{-\xi(x, k)} .
$$

Thus the adjoint wave function has a pole at $k=p$ while the wave function $w$ has a pole at $k=\mathrm{q}$. 


\section{§ 3. Fredholm Determinants and Minors}

Since we are operating on a semi-infinite interval, we must impose decay conditions of the kernel $F$ so that the trace and Fredholm determinant of the truncated integral operator $F_{a}$ are finite. We impose a condition on the behavior of the kernel $F(x, z)$ as $x_{1}, z_{1}$ tend to infinity. (Since we are concerned only with the decay of $F$ in the variables $x_{1}$ and $z_{1}$ we regard the other variables as fixed here.) For $1 / 2<\nu \leq 1$ we require that

$$
\begin{aligned}
& \sup _{a \leq s, t<\infty}|F(s, t) \| 1+s|^{v}|1+t|^{0}<\infty \\
& a-\infty
\end{aligned}
$$

for all $\mathrm{a}_{0}$ Then the truncated integral operator $F_{a}$ is of trace class and its Fredholm determinant is well defined. (cf. [12], Appendix A) Under these conditions the GLM equation is amenable to a variant of Fredholm's theory of integral equations. In this section we summarize the basic facts about Fredholm determinants and minors which will be needed in the sequel. Two convenient references are Riesz and Nagy [16], and Smithies [20].

Equation (2.3) can be interpreted as a kind of resolvent equation for the kernel $K_{+}$given the kernel $F$ on the interval $\left(x_{1}, \infty\right)$. There is a slight anomaly, in that the lower limit of integration, $x_{1}$, is one of the variables; so that the GLM is not strictly in the form of a resolvent equation for a Fredholm integral equation. The usual arguments in Fredholm's theory, however, can be carried through unaltered.

The Fredholm determinant for the GLM equation (2.3) on the interval $\left(x_{1}, \infty\right)$ is

$$
D(x)=\sum_{n=0}^{\infty} \frac{1}{n !} \int_{x_{1}}^{\infty} \cdots \int_{x_{1}}^{\infty} F\left[\begin{array}{l}
\eta_{1} \cdots \eta_{n} \\
\eta_{1} \cdots \eta_{n}
\end{array}\right] d y_{1} \cdots d y_{n}
$$

where

$$
F\left[\begin{array}{l}
\xi_{1} \cdots \xi_{n} \\
\eta_{1} \cdots \circ \eta_{n}
\end{array}\right]=\operatorname{det}|| F\left(\xi_{j}, \eta_{k}\right) \|_{1 \leq j, k \leq n} \cdot
$$

The leading term is simply 1 . The variables $\eta_{j}$ are hierarchy variables $: \eta_{j}=\left(y_{j}, x_{2}, x_{3}, \ldots\right)$.

We are going to see presently that $D(x)$ gives the $\tau$ function for 
the KP hierarchy. $D(x)$ is the Fredholm determinant of the truncated integral operator

$$
F_{\left(x_{1}\right)} \Psi(x)=\int_{x_{1}}^{\infty} F(x, z) \Psi\left(z_{1}\right) d z_{1}
$$

The Fredholm "minor" for (2.3) over $\left(x_{1}, \infty\right)$ is

$$
D(x, z)=-\sum_{n=0}^{\infty} \frac{1}{n !} \int_{x_{1}}^{\infty} \cdots \int_{x_{1}}^{\infty} F\left[\begin{array}{l}
x, \eta_{1} \cdots \eta_{n} \\
z, \eta_{1} \cdots \eta_{n}
\end{array}\right] d y_{1} \cdots d y_{n} .
$$

The kernel $K_{+}$in the GLM equation is then

$$
K_{+}(x, z)=\frac{D(x, z)}{D(x)}
$$

The formula (3.3) is Gramer s rule for the GLM equation. It can be checked by direct verification, using exactly the same arguements as those used in the case of the resolvent equation, that $K_{+}$defined by $(3.1,3.2,3.3)$ is a solution of the GLM equation (cf. for example, [16], p. 174).

Hirota introduced the transformation $u=2(\log \tau)_{x x}$ for the $\mathrm{KdV}$ and the KP equations, and showed that $\tau$ satisfied certain bilinear differential equations. The transformation is suggested by the multisoliton solution of the $\mathrm{KdV}$ equation. The multisoliton solution can be obtained by solving the GLM equation where, for the $\mathrm{KdV}$ equation, $F(x, z)=F(x+z)$ and

$$
F(s)=\sum_{j=1}^{N} c_{n} e^{-\omega_{n} s}
$$

The dependence on $t$ and the higher order hierarchy variables $\mathbf{x}_{2 \jmath+1}$ is implicit in $c_{n}$. The GLM equation reduces to an algebraic system, and it is found that

$$
u(x)=2 \frac{d^{2}}{d x^{2}} \log \operatorname{det}|| 1+A \|
$$

where $A$ is the matrix of coefficients of the algebraic system. (cf. for example, the account in [9].) In the case of the $N$-soliton solution, the $\tau$ function is precisely the determinant $\operatorname{det}\|1+A\|$; in the general case $\operatorname{det}\|1+A\|$ is replaced by the Fredholm determinant (3.1) ( $[10]$, $[13,14]$ ).

Similarly, in the case of the $\mathrm{KP}$ equation, we have: 
Theorem 3.1. Let a solution of the KP hierarchy be generated by the kernel $F(x, z)$ which satisfies the differential equations (2.4). Then the potential $u$ in the isospectral operator $\partial / \partial y-D^{2}-u$ is given by

$$
u(x)=2\left(\frac{\partial^{2}}{\partial x_{1}^{2}}\right) \log D(x)
$$

where $D(x)$ denotes the Fredholm determinant det $\left(1+F_{(x)}\right)$ as given by the series (3.1).

This theorem allows us to identify $D(x)$ with the $\tau$ function. The fact that $F$ satisfies equations (2.4) means that the commutation relations (1.3) for the operators $B_{n}$ are satisfied. It follows that their coefficients satisfy the equations of the KP hierarchy; and, in particular, $u$ satisfies the KP equation. The other coefficients of the operators $B_{n}$ in the hierarchy can be obtained as derivatives of log $D(x)$ following the same procedure as in [4].

We prove Theorem 3.1 by showing that

$$
K_{+}(x, x)=\frac{\partial}{\partial x_{1}} \log D(x) 。
$$

The result then follows from the first equation in (2.2). From (3.3) it suffices to prove that

$$
D(x, x)=\frac{\partial}{\partial x_{1}} D(x) .
$$

We do this by differentiating the series (3.1) with respect to $x_{1}$ to obtain (3.2). In this calculation none of the variables $x_{2}, x_{3}, \ldots$ plays a role, so we may ignore the dependence on these variables. The derivative of the $n$-fold integral

$$
\frac{\partial}{\partial x_{1}} \frac{1}{n !} \int_{x_{1}}^{\infty} \cdots \int_{x_{1}}^{\infty} F\left[\begin{array}{l}
y_{1}, \ldots y_{n} \\
y_{1}, \ldots y_{n}
\end{array}\right] d y_{1} \cdots d y_{n}
$$

is a sum of $n$ terms, one for each of the integrals. These are seen to be equal by using the fact that the determinants

$$
F\left[\begin{array}{l}
x_{1} \cdots x_{n} \\
y_{1} \cdots y_{n}
\end{array}\right]=\operatorname{det}\left\|F\left(x_{i}, y_{j}\right)\right\|
$$

are unchanged under the transposition of a pair

$$
\left[\begin{array}{l}
x_{i} \\
y_{i}
\end{array}\right] \text { and }\left[\begin{array}{l}
x_{j} \\
y_{j}
\end{array}\right]
$$


and by making the appropriate changes of variables of integration in each of the terms. So we obtain

$$
-\frac{1}{(n-1) !} \int_{x_{1}}^{\infty} \cdots \int_{x_{1}}^{\infty} F\left[\begin{array}{l}
x, y_{2}, \ldots, y_{n} \\
x, y_{2}, \ldots, y^{n}
\end{array}\right] d y_{2} \cdots d y_{n} \text {. }
$$

We thus obtain the $(n-1)^{\text {st }}$ term in the series (3.2) by differentiating the $n^{\text {th }}$ term of the series (3.1) with respect to $x_{1}$. This proves Theorem 3.1.

The $\tau$ function for the $N$-soliton solution of the KP hierarchy is easily obtained by standard methods. Taking $F$ as given in (2.7), we obtain a system of algebraic equations for the kernel $K_{+}$ from (2.3). Just as in the case of the $\mathrm{KdV}$ equation, the $\tau$ function for the $\mathrm{N}$-soliton solution of the KP hierarchy is given by

$$
\tau(x)=\operatorname{det}\left\|\delta_{j k}+\frac{a_{k}}{q_{j}-p_{k}} e^{\xi\left(x, p_{k}\right)-\xi\left(x, q_{j}\right)}\right\|
$$

and the $N$-soliton solution of the $\mathrm{KP}$ equation is $u=2 \partial_{x}^{2}(\log \tau)$.

Some special cases will be discussed in $\S 6$. From the relation $u=2 \partial_{x}^{2}(\log \tau)$, it is clear that zeroes of $\tau$ lead to singularities, in fact poles, in $u$. We give some conditions in $\$ 6$ which guarantee the positivity of $\tau$.

The concrete representation of the $\tau$ function by the series (3.1) makes it possible to investigate the validity of the formalism in the case of the general initial value problem for the KP hierarchy, at least in the case of KP I, where the initial value problem can be treated by a local Riemann-Hilbert problem. The fact that we are dealing with an infinite hierarchy means that it is natural to require $\tau$ to be $C^{\infty}$ in all its variables; for the coefficients of the differential operators $B_{n}$ are obtained as derivatives of all orders of $\log \tau$.

The phase function $\xi(x, k)$ is analytic in all its variables if, for example, the hierarchy variables satisfy the condition

$$
\lim _{n \rightarrow \infty} \sup \left|x_{n}\right|^{1 / n}=0 .
$$

Let us denote the set of such hierarchy variables by $\boldsymbol{H}$. For example, $\boldsymbol{H}$ contains the set of all $x$ in which all but a finite number of the $x_{n}$ vanish. We shall always assume $x \boldsymbol{H} \boldsymbol{H}$.

If the integration in (2.6) is taken over a region in which 
$\operatorname{Re} p(s) \leq-\delta<0<\delta \leq \operatorname{Re} q(s)$, then $F$ is analytic in $x$ and $z$, and decays exponentially to zero as $z_{1} \rightarrow \infty$. Under these conditions it is easily verified that $\tau$ is defined and analytic for all $x \varepsilon H$.

As we noted in $\S 1$, there are two distinct cases of the $\mathbb{K P}$ hierarchy: KP I in which all $x_{2 j+1}$ are real and all $x_{2}$ are imaginary; and KP II in which all the $x_{j}$ are real. The solution of the initial value problem for KP I can be treated by a nonlocal RiemannHilbert problem (cf. [1], [25]) which is equivalent to the GLM equation. The solution of the initial value problem for KP I using the GLM equation has been discussed by Manakov [8]. For general initial values, the kernel $F$ contains a term of the form

$$
F(x, z)=\int_{-\infty}^{\infty} \int_{-\infty}^{\infty} e^{\xi(x, i p)-\xi(z, i q)} f(p, q) d p d q
$$

that is, where the measure, $\mu$ in $(2.6)$ has support only on the imaginary $p$ and $q$ axes. The density $f$ is the analog of the reflection coefficient in the $\mathrm{KdV}$ equation. For $\mathrm{KP} I$ the argument in the exponential term in this integral is purely imaginary, and $F$ is a kind of Fourier transform of $f(p, q)$.

As long as $x \notin \mathbb{Z}$ the usual arguments of Fourier analysis apply for the KP I case. For example, let $\mathbb{S}$ denote the class of functions $f$ for which

$$
\sup _{p, q}\left|p^{m} q^{n} \frac{\partial^{\mu+v}}{\partial p^{\mu} \partial q^{v}} f(p, q)\right|<+\infty 。
$$

Then it is easily seen that $F$ also belongs to this class as a function of $x_{1}$ and $z_{1}$; and furthermore that $F$ is differentiable with respect to all the hierarchy variables as long as $x \in \mathbb{H}$.

Theorem 3.2. Let the density $f(p, q)$ belong to the class $\mathbb{S}$; then $\tau$ is $C^{\infty}$.

Proof. Let $a_{1}, a_{2}, \ldots, a_{n}$ be column vectors in $C^{n}$, and let $A=\| a_{1}, a_{2}, \ldots$ $a_{n} \|$. Hadamard's inequality states (cf. [16], p. 176), $|\operatorname{det} A| \leq|| a_{1}\|\| a_{2} \|$ $\ldots\left\|a_{n}\right\|$, where $\left\|a_{\|}\right\|$is the Euclidean norm of the column vector $a_{j}$. An immediate consequence of this inequality is that if each of the entries of the matrix $A$ is bounded in absolute value by $m$, then $\left\|a_{3}\right\| \leq n^{1 / 2} m$, and $|\operatorname{det} A| \leq m^{n} n^{n / 2}$. Now suppose the vectors $a_{j}$ depend on a set of variables $x_{0}, x_{1}, x_{2}, \ldots$ and let 


$$
D^{\alpha}=\frac{\partial^{|\alpha|}}{\partial x_{0}^{\alpha} \partial^{\alpha} x_{1}^{\alpha} \ldots}
$$

where $\alpha=\left(\alpha_{0}, \alpha_{1}, \ldots\right)$ is a sequence of non-negative integers, with $|\alpha|=\alpha_{0}+\alpha_{1}+\ldots<\infty$. Then $D^{\alpha}$ det $A$ is a sum of $n^{|\alpha|}$ determinants, by Leibniz's rule for determinants. Let us assume that $\left|D^{\alpha} a,(x)\right| \leq$ $m_{j}(x)$ for all $|\alpha| \leq k$ for some set of functions $m_{j}(x)$ on a domain of the variables $x$ and some fixed integer $k$. An immediate consequence of Hadamard's inequality is that

$$
\left|D^{\alpha} \operatorname{det} A\right| \leq n^{|\alpha|+n / 2} m_{1}(x) m_{2}(x) \ldots m_{n}(x) \text { for }|\alpha| \leq k \text {. }
$$

Now we apply these considerations to the series (3.1) for the Fredholm determinant. Since $f(p, q)$ is rapidly decreasing, $F(x, z)$ is in the class $\boldsymbol{S}$. We consider $z_{1}$ to be the variable $x_{0}$. Since $F$ is in $\boldsymbol{S}$ we have the uniform estimates $\left|D^{\alpha} F(x, z)\right| \leq m_{q}(z)$ for $x \varepsilon \boldsymbol{H}$, $|\alpha| \leq q, z_{1} \geq x_{1}$. The function $m_{q}\left(z_{1}\right)$ is furthermore integrable on the interval $\left(x_{1}, \infty\right)$. By (3.4)

$$
D^{\alpha} F\left[\begin{array}{l}
\eta_{1}, \eta_{2} \cdots \eta_{n} \\
\eta_{1}, \eta_{2} \cdots \eta_{n}
\end{array}\right] \leq n^{|\alpha|+n / 2}\left(m_{q}\left(y_{1}\right) m_{q}\left(y_{2}\right) \cdots m_{q}\left(y_{n}\right)\right)
$$

where $\eta_{j}=\left(y_{j}, x_{2}, \ldots\right)$.

When $\alpha_{1}=0$ the series for $D^{\alpha} D(x)$ is obtained by differentiating under the $n$-fold integrals; it is dominated by the series

$$
\sum_{n=0}^{\infty} \frac{1}{n !} n^{|\alpha|+n / 2} M_{q}^{n}(x)
$$

where

$$
M_{q}(x)=\int_{x_{1}}^{\infty} m_{q}(y) d y_{1}
$$

In particular the series (3.1) itself is convergent. When the derivative $D^{\alpha}$ contains differentiations with respect to $x_{1}$, the situation is slightly more complicated; but the convergence proof is essentially the same. We omit the details.

\section{§4. The Bilinear Identity}

We begin by stating the bilinear identity for the KP hierarchy as given in $[5,16]$. Let us recall $(\S 2)$ that a wave function $w$ and 
adjoint wave function $w^{*}$ for the KP hierarchy satisfy the equations

$$
\left(\frac{\partial}{\partial x_{n}}-B_{n}\right) w=0 \quad\left(\frac{\partial}{\partial x_{n}}+B_{n}^{*}\right) w^{*}=0
$$

where the hierarchies $\left\{B_{n}\right\}$ and $\left\{B_{n}^{*}\right\}$ are obtained by dressing the operators

$$
\frac{\partial}{\partial x_{n}}-D^{n} \text { and } \quad \frac{\partial}{\partial x_{n}}+(-1)^{n} D^{n} \quad n=2,3, \ldots
$$

respectively.

In what follows we shall assume that $w$ and $w^{*}$ have the representations, $w=\left(1+K_{+}\right) e^{\xi(x, k)}$, and $w^{*}=\left(1+K_{+}^{t}\right)^{-1} e^{-\xi(x, k)}$. For a discussion of the wave functions of the KP equation and their properties, see $[1,8]$. If $w$ and $w^{*}$ have the representations $w=\left(1+K_{+}\right) e^{\xi(x, k)}$, etc. then they are analytic for $k$ in some left half plane, and have the asymptotic behavior

$$
w(x, k) e^{-\xi(x, k)}-1=O\left(\frac{1}{k}\right) \quad \text { as } k \rightarrow \infty \text { in } R e \quad k<0
$$

and similarly for $w^{*}$. Conversely, any wave function $w$ which is analytic in $R e \quad k<0$ and satisfies (4.2) has the integral representations above, by the Paley-Wiener theorem.

The bilinear identity is then:

Theorem 4.1. Let $w(x, k)$ and $w^{*}(x, k)$ be the wave function and adjoint wave function for the KP and adjoint KP hierarchies, analytic in $k$ in some left half plane, $C^{\infty}$ in the variables $x_{\text {, }}$, and satisfying (4.2). Then

$$
\int_{C} w(x, k) w^{*}\left(x^{\prime}, k\right) d k=0 \quad \text { for all } x, x^{\prime}
$$

where $C$ is a contour that runs parallel to the entire imaginary axis in the complex plane, and $x, x^{\prime}$ are the hierarchy variables.

Conversely, let $w(x, k)$ and $w^{*}(x, k)$ be analytic in $k$ on some left half plane, satisfying the asymptotic conditions (4.2), and infinitely differentiable in each of the variables $x$,. Then $w$ and $w^{*}$ are the wave functions for some KP hierarchy and its adjoint.

The second statement of the theorem means that there exists a family of differential operators $B_{n}$ and $B_{n}^{*}$ for which the equations (4.1) are satisfied. The construction of these operators follows 
readily once the Volterra dressing operators $K_{ \pm}$are obtained. Given $w$ and $w^{*}$ satisfying the conditions of the theorem, their representation in terms of the Volterra integral operators $K_{ \pm}$follows from the Paley-Wiener theorem.

This theorem was stated and proved in [4] for the case where $w$ and $w^{*}$ have convergent Laurent expansions in $1 / k$ for sufficiently large values of $k$. This holds, in particular, for multi-soliton solutions of the KP hierarchy. The contour $C$ was taken to be a closed contour in the complex plane enclosing the singularities of $w$ and $w^{*}$.

The bilinear identity is based on the following lemma :

Lemma 4.2. Let $P$ and $Q$ be respectively upper and lower Volterra integral operators. Then

$$
\begin{aligned}
\frac{1}{2 \pi i} \int_{C} & (1+P) e^{k x}(1+Q) e^{-k y} d k \\
= & \begin{cases}P(x, y)+Q(y, x)+\int_{x}^{y} P(x, t) Q(y, t) d t & x<y \\
0 & x>y\end{cases}
\end{aligned}
$$

where $C$ is a contour that runs parallel to the entire imaginary axis.

In its application to the bilinear identity we take $w=(1+P) e^{\xi(x, k)}$ and $w^{*}=(1+Q) e^{-\xi(x, k)}$. The bilinear identity for $w$ and $w^{*}$ implies that $(1+P)\left(1+Q^{t}\right)=1$, hence that $(1+Q)=\left(1+K_{+}^{t}\right)^{-1}$. This relationship allows one to show that $w$ and $w^{*}$ are in fact wave function and adjoint wave function for the $\mathrm{KP}$ and $\mathrm{KP}^{*}$ hierarchies. Once Lemma 4.2 is established the bilinear identity is proved along the same lines to be found in [4], pp 59, 60 .

In [4] Lemma 4.2 was stated for pseudo-differential operators $P$ and $Q$, and it was assumed that these operators, applied to $e^{ \pm \xi(x, k)}$ resulted in wave functions convergent in $1 / k$ for sufficiently large $k$. The proof below extends the validity of Lemma 4.2 to wave functions analytic in some left half plane. Our proof is based on the inversion theorem for the Laplace transform, and on the convolution theorem for the Laplace transform.

Proof of Lemma 4.2. As far as the proof of the lemma goes, we need only deal with a single scalar variable, and the higher order 
hierarchy variables play no role. Multiplying out the two factors in the integrand, we obtain four terms. The first term vanishes,

$$
\frac{1}{2 \pi i} \int_{C} e^{k(x-y)} d k=0
$$

by closing the contour $C$ in the left or right half plane according as $x-y$ is positive or negative. The second term is

$$
\frac{1}{2 \pi i} \int_{C} e^{-k y} \int_{x}^{\infty} P\left(x, x^{\prime}\right) e^{k x^{\prime}} d x^{\prime}=\frac{1}{2 \pi i} \int_{C} e^{k(x-y)} \int_{0}^{\infty} P(x, x+z) e^{k z} d z_{。}
$$

We may interpret this as a Laplace transform and its inverse (say, the Laplace transform evaluated at $-k$ ). The inversion theorem for the Laplace transform then gives

$$
P(x, x+(y-x))=P(x, y)
$$

for this term. Similarly, the term involving only $Q$ can be shown to be $Q(y, x)$. The final term can be written

$$
\frac{1}{2 \pi i} \int_{C}\left\{\int_{x}^{\infty} P(x, z) e^{k z} d z\right\}\left\{\int_{-\infty}^{y} Q\left(y, z^{\prime}\right) e^{-k z^{\prime}} d z^{\prime}\right\} d k .
$$

By changing variables and using $\sigma=z-x$ and $\sigma^{\prime}=y-z^{\prime}$, we get

$$
\begin{aligned}
& \frac{1}{2 \pi i} \int_{C}\left\{\int_{0}^{\infty} P(x, \sigma+x) e^{k(\sigma+x)} d \sigma\right\}\left\{\int_{0}^{\infty} Q\left(y, y-\sigma^{\prime}\right) e^{k\left(\sigma^{\prime}-y\right)} d \sigma^{\prime}\right\} d k \\
& \quad=\frac{1}{2 \pi i} \int_{C} e^{-k(y-x)} H_{+}(x, k) H_{-}(y, k) d k
\end{aligned}
$$

where

$$
H_{+}(x, k)=\int_{0}^{\infty} e^{k t} P(x, x+t) d t \text { and } H_{-}(y, k)=\int_{0}^{\infty} e^{k t} Q(y, y-t) d t .
$$

By the Laplace inversion theorem and the convolution theorem for the Laplace transform the preceeding integral reduces to

$$
\int_{x}^{y} P(x, t) Q(y, t) d t
$$

and the lemma is proved.

Hirota (cf. his review article [6]) introduced the transformation $u=2(\log \tau)_{x x}$ and showed that $\tau$ satisfied a certain bilinear differential equation. Let $f$ and $g$ be functions of $x$ and $t$ and define

$$
D_{t}^{n} D_{x}^{m} f \cdot g=\left.\left(\partial / \partial t-\partial / \partial t^{\prime}\right)^{n}\left(\partial / \partial x-\partial / \partial x^{\prime}\right)^{m} f(x, t) g\left(x^{\prime}, t^{\prime}\right)\right|_{x=x^{\prime}, t=t^{\prime}}
$$

For example, $D_{t} f \cdot g=f_{t} g-f g_{t} ; D_{x}^{2} f \cdot g=f_{x x} g-2 f_{x} g_{x}+f g_{x x}$; etc. Then the 
$\mathrm{KdV}$ equation can be rewritten in the bilinear form

$$
D_{x}\left(D_{x}^{3}-4 D_{t}\right) \tau \cdot \tau=0
$$

where $u=2(\log \tau)_{x x}$. Under the same transformation he showed that the $\mathrm{KP}$ equation has the bilinear form

$$
\left(D_{x}^{4}+3 D_{y}^{2}-4 D_{x t}\right) \tau \cdot \tau=0 .
$$

In [4] the bilinear identity leads to a generating function for an infinite hierarchy of bilinear differential equations for the $\tau$ function. That proof requires the relationship of the $\tau$ function to the wave function via the vertex operator. We discuss that relationship in the next section.

\section{§5. The Vertex Operator}

In principle, the $\tau$ function is supposed to carry all the information about the solutions of the hierarchy. All the coefficients of the operators $B_{n}$ may be obtained as derivatives of the $\tau$ function with respect to the hierarchy variables $x_{n}$. In addition, there is a simple relationship between the wave function $w(x, k)$ and the $\tau$ function. Let the operator $G(k)$ be defined by

$$
G(k) \tau(x)=\tau\left(x_{1}-\frac{1}{k}, x_{2}-\frac{1}{2 k^{2}}, x_{3}-\frac{1}{3 k^{3}}, \ldots\right) ;
$$

then the vertex operator $X(k)$ is given by $X(k)=e^{\xi(x, k)} G(k)[4]$.

Theorem 5.1. Let $F$ be given by (2.6), where Re $p(s)<-\delta<0$ $<\delta<\operatorname{Re} q\left(s^{\prime}\right)$ for all $s, s^{\prime}$ and let $\mu$ be such that the Fredholm determinant of $F_{x}$ is always defined. Then the following relationship holds between the vertex operator, the wave function $w(x, k)$ and the $\tau$ function for Re $k<0$ :

$$
X(k) \tau(x)=w(x, k) \tau(x), \quad \text { Re } k<0 .
$$

Proof : We present a proof of this result here based directly on the representation of $\tau$ as a Fredholm determinant. From (3.3) and the representation $w=\left(1+K_{+}\right) e^{\xi(x, k)}$ we see that (5.1) can be written in the form 


$$
\frac{G(k) \tau(x)}{\tau(x)}=w(x, k)=1+\int_{x_{1}}^{\infty} \frac{D\left(x, z_{1}\right)}{\tau(x)} e^{\xi(z-x, k)} d z_{1}
$$

Since $x$ and $z$ coincide in all the higher order variables, only the term $e^{k(z-x)}$ occurs in the integrand, and (5. $\left.1^{\prime}\right)$ reduces to

$$
(G(k)-1) \tau(x)=\int_{x_{1}}^{\infty} D\left(x, z_{1}\right) e^{k\left(z_{1}-x_{1}\right)} d z_{1}
$$

We shall derive (5.2) for a general $F$ given by (2.6) under the conditions on $p$ and $q$ in the theorem. From (3.1) the general term in the series for $\tau$ is

$$
\frac{1}{n !} \int_{x_{1}}^{\infty} \cdots \int_{x_{1}}^{\infty} \operatorname{det}\left\|F\left(\eta_{j}, \eta_{k}\right)\right\| d y_{1} \cdots d y_{n}
$$

where $\eta_{j}=\left(y_{j}, x_{2}, x_{3} \ldots\right)$. Applying the vertex operator to this general term we find that

$$
\begin{aligned}
& G(k) \int_{x_{1}}^{\infty} \cdots \int_{x_{1}}^{\infty} \operatorname{det}\left\|F\left(\eta_{j}, \eta_{k}\right)\right\| d y_{1} \cdots d y_{n} \\
& =\int_{x_{1}}^{\infty} \cdots \int_{x_{1}}^{\infty} \operatorname{det}\left\|G(k) F\left(\eta_{j}, \eta_{k}\right)\right\| d y_{1} \cdots d y_{n} .
\end{aligned}
$$

In fact, the effect of $G(k)$ is to shift the lower limits of integration in the integral to $x_{1}-1 / k$ and from $x_{n}$ to $x_{n}-1 /\left(n k^{n}\right)$ inside the integrand. The result above is obtained by changing the variable of integration : $y_{j} \rightarrow y_{j}+1 / k$.

Now

$$
\begin{aligned}
\operatorname{det}\left\|F\left(\eta_{j}, \eta_{k}\right)\right\| & =\sum_{\pi \in S_{n}}(-1)^{\pi} F\left(\eta_{1}, \eta_{\pi(1)}\right) F\left(\eta_{2}, \eta_{\pi(2)}\right) \ldots F\left(\eta_{n}, \eta_{\pi(n)}\right) \\
& =\sum_{\pi \in S_{n}}(-1)^{\pi} \prod_{j=1}^{n} \int \exp \left\{\xi\left(\eta_{j}, p_{j}\right)-\xi\left(\eta_{\pi(j)}, q_{j}\right) d \mu\left(s_{j}\right)\right\}
\end{aligned}
$$

where $p_{j}=p\left(s_{j}\right)$ and $q_{j}=q\left(s_{j}\right)$, and $S_{n}$ is the permutation group on $\{1, \ldots n\}$. Now

$$
G(k) e^{\xi(x, p)}=e^{\xi(x, p)}(1-p / k)
$$

and applying this to (5.3) we get

$$
\begin{aligned}
& G(k) \operatorname{det}\left\|F\left(\eta_{j}, \eta_{k}\right)\right\| \\
& =\sum_{\pi \varepsilon S_{n}}(-1)^{\pi} \prod_{j=1}^{n} \int \exp \left\{\xi\left(\eta_{j}, p_{j}\right)-\xi\left(\eta_{\pi(j)}, q_{j}\right)\right\} \frac{k-p_{j}}{k-q_{j}} d \mu\left(s_{j}\right) .
\end{aligned}
$$

Integrating with respect to $y_{1} \cdots y_{n}$ and interchanging the order of in- 
tegration, we get

$$
\begin{aligned}
& \int_{x_{1}}^{\infty} \cdots \int_{x_{1}}^{\infty} \sum_{\pi \varepsilon S_{n}}(-1)^{\pi} \prod_{j=1}^{n} \int \exp \left\{\xi\left(\eta_{j}, p_{j}\right)-\xi\left(\eta_{\pi(j)}, q_{j}\right) \frac{k-p_{j}}{k-q_{j}} d \mu d y\right. \\
& =\sum_{\pi \in S_{n}}(-1)^{n} \int \cdots \int d \mu\left\{\prod_{j=1}^{n} \frac{k-p_{j}}{k-q_{j}} \int_{x_{1}}^{\infty} \exp \left\{\xi\left(\eta_{j}, p_{j}\right)-\xi\left(\eta_{j}, q_{\pi^{-1}(\jmath)}\right)\right\} d y_{j}\right\}
\end{aligned}
$$

where $d \mu=d \mu\left(s_{1}\right) \ldots d \mu\left(s_{n}\right)$ and $d y=d y_{1} \ldots d y_{n}$. The integrations with respect to the $y$, variables are easily carried out, provided $R e p_{j}-q_{k}$ $<0$ for all $j$ and $k$; and we obtain

$$
\begin{aligned}
& \sum_{\pi \varepsilon S_{n}}(-1)^{\pi} \int \cdots \int d \mu \prod_{j=1}^{n} \exp \left\{\xi\left(x, p_{j}\right)-\xi\left(x, q_{\pi^{-1}(j)}\right)\right\} \frac{k-p_{j}}{k-q_{j}} \cdot \frac{1}{q_{\pi^{-1}(j)}-p_{j}} \\
& =\int \cdots \int E_{n}(x, s) \Lambda_{n} D_{n} d \mu
\end{aligned}
$$

where,

$$
\begin{aligned}
& E_{n}(x, s)=\exp \left\{\xi\left(x, p_{1}\right)+\cdots \xi\left(x, p_{n}\right)-\xi\left(x, q_{1}\right)-\cdots-\xi\left(x, q_{n}\right)\right\} \\
& \Lambda_{n}=\prod_{j=1}^{n} \frac{k-p_{j}}{k-q_{j}} \quad D_{n}=\operatorname{det}\left\|\frac{1}{q_{j}-p_{k}}\right\|_{1 \leq j, k \leq n^{\bullet}}
\end{aligned}
$$

The other term is treated similarly, and the general term of $(G(k)$ $-1) \tau(x)$ is

$$
\frac{1}{n !} \int \cdots \int E_{n}\left(\Lambda_{n}-1\right) D_{n} d \mu_{0}
$$

Turning to the right side of (5.2), we need to calculate the $(n+1)$ fold integral

$$
\frac{1}{n !} \int_{x_{1}}^{\infty} \cdots \int_{x_{1}}^{\infty} F\left[\begin{array}{l}
x, \eta_{1} \cdots \eta_{n} \\
z, \eta_{1} \cdots \eta_{n}
\end{array}\right] e^{k\left(z_{1}-x_{1}\right)} d z_{1} d y_{1} \cdots d y_{n}
$$

where $\eta_{j}=\left(y_{j}, x_{2}, x_{3} \ldots\right)$. Let us put $\eta_{0}=z=\left(z_{1}, x_{2}, \ldots\right)$. Substituting in for $F$ from (2.6) we get

$$
\begin{aligned}
& \frac{1}{n !} \int d \mu \sum_{\pi \varepsilon S_{n+1}}(-1)^{\pi} \int_{x_{1}}^{\infty} \cdots \int_{x_{1}}^{\infty} e^{k\left(z_{1}-x_{1}\right)} \exp \left\{\xi\left(x, p_{0}\right) .\right. \\
& \left.\quad+\cdots+\xi\left(x, p_{n}\right)-\xi\left(\eta_{\pi(0)}, q_{0}\right)-\cdots-\xi\left(\eta_{\pi(n)}, q_{n}\right)\right\} d y .
\end{aligned}
$$

In this integration, $d \mu=d \mu\left(s_{0}\right) \ldots d \mu\left(s_{n}\right)$, and the $\mu$ integration is an $(n+1)$ fold integral ; and $d y=d z_{1} d y_{1} \ldots d y_{n}$. The $(n+1)$ fold integration with respect to the $y$ variables reduces to a product of $(n+1)$ integrals : 


$$
\begin{aligned}
& e^{\xi\left(x, p_{0}\right)-k x_{1}} \int_{x_{1}}^{\infty} \cdots \int_{x_{1}}^{\infty} \exp \left\{k z_{1}-\xi\left(z, q_{\pi^{-1}(0)}\right)\right\} \prod_{j=1}^{n} \exp \left\{\xi\left(\eta_{j}, p_{j}\right)-\xi\left(\eta_{j}, q_{\pi^{-1}(j)}\right\} d y\right. \\
& =\exp \left\{\xi\left(x, p_{0}\right)-\xi(x, k)\right\} \int_{x_{1}}^{\infty} \exp \{\xi(z, k) \\
& \left.\quad-\xi\left(z, q_{\pi^{-1}(0)}\right)\right\} d z_{1} \prod_{j=1}^{n} \int_{x_{1}}^{\infty} \exp \left\{\xi\left(\eta_{j}, p_{j}\right)-\xi\left(\eta_{j}, q_{\pi^{-1}(j)}\right)\right\} d y_{j}
\end{aligned}
$$

Each of these integrations can be carried out, and the result is

$$
\left\{\prod_{j=1}^{n} \exp \left\{\xi\left(x, p_{j}\right)-\xi\left(x, q_{j}\right)\right\} \frac{1}{\left(q_{\pi^{-1}(0)}-k\right)\left(q_{\pi^{-1}(1)}-p_{1}\right) \cdots}\right.
$$

Summing over $\pi$ in $S_{n+1}$ in $(5.5)$ we get $E_{0} E_{n} D_{n+1,0}$, where

$$
E_{0}=\exp \left\{\xi\left(x, p_{0}\right)-\xi\left(x, q_{0}\right)\right\}
$$

$E_{n}$ is as given above, and $D_{n+1,0}$ is the determinant obtained from $D_{n+1}$ by replacing $p_{0}$ by $k$ :

$$
D_{n+1,0}=\operatorname{det}\left[\begin{array}{cccc}
\frac{1}{q_{0}-k} \frac{1}{q_{0}-p_{1}} & \ldots & \ldots & \frac{1}{q_{0}-p_{n}} \\
\frac{1}{q_{1}-k} \frac{1}{q_{1}-p_{1}} & \ldots & \ldots & \frac{1}{q_{1}-p_{n}} \\
\frac{1}{q_{n}-k} \frac{1}{q_{n}-p_{1}} & \ldots & \ldots & \frac{1}{q_{n}-p_{n}}
\end{array}\right] .
$$

By relabelling the variables in the obvious way, we reduce the identity (5.2) to

$$
\frac{1}{n !} \int \cdots \int E_{n} D_{n}\left(\Lambda_{n}-1\right) d \mu=\frac{1}{(n-1) !} \int \cdots \int E_{n} D_{n, 1} d \mu
$$

where the variables of integration run over $s_{1} \ldots s_{n}$, and $D_{n, 1}$ is the determinant obtained by replacing $p_{1}$ by $k$ in $D_{n}$ :

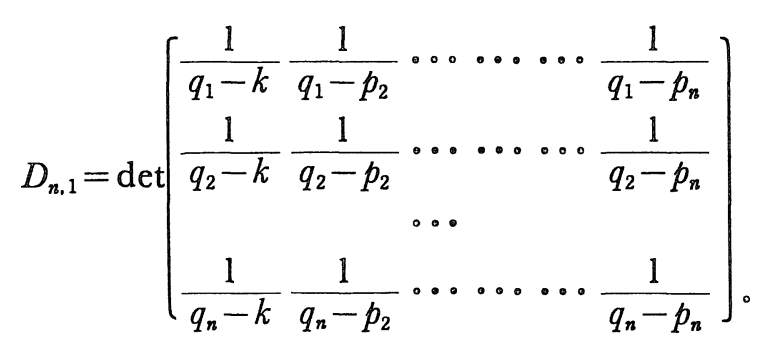

Now there is a certain asymmetry in the identity (5.6) as it is stated : namely, $p_{1}$ is missing in $D_{n, 1}$. On the other hand $E_{n}$ is sym- 
metric in the variables $s_{1} \ldots s_{n}$. If we interchange $s_{1}$ and $s_{j}$ in the right hand side of (5.6) then $D_{n, 1}$ is transformed into $D_{n, j}$. Therefore the right side of (5.6) can be written as a sum of $n$ terms, and (5.1) reduces to showing

$$
D_{n}\left(\Lambda_{n}-1\right)=D_{n, 1}+D_{n, 2}+\cdots+D_{n, n},
$$

where $D_{n, j}$ is obtained by replacing $p_{j}$ by $k$ in the determinant for $D_{n}$.

The determinants $D_{n}$ and $D_{n, m}$ can be evaluated by using the identity (cf. [11], p.98)

$$
D_{n}=\operatorname{det}|| \frac{1}{q_{j}-p_{l}} \|=\frac{\prod_{j>l}\left(q_{j}-q_{l}\right)\left(p_{l}-p_{j}\right)}{\prod_{j, l=1}^{n}\left(q_{j}-p_{l}\right)}
$$

for $D_{n}$, and a similar expression for $D_{n, m}$. Cancelling out common factors, we find that

$$
\begin{aligned}
\frac{D_{n, m}}{D_{n}} & =\prod_{j=1}^{n} \frac{p_{j}-k}{q_{j}-k}\left(\frac{q_{m}-p_{m}}{p_{m}-k}\right)_{j \neq m} \frac{q_{j}-p_{m}}{p_{j}-p_{m}} \\
& =\Lambda_{n} \frac{1}{\left(p_{m}-k\right)} \frac{\prod_{j=1}^{n}\left(q_{j}-p_{m}\right)}{\prod_{j \neq m}\left(p_{j}-p_{m}\right)}=\Lambda_{n} \frac{1}{\left(p_{m}-k\right)} \frac{Q\left(p_{m}\right)}{P^{\prime}\left(p_{m}\right)}
\end{aligned}
$$

where

$$
P(k)=\prod_{j=1}^{n}\left(p_{j}-k\right) \quad \text { and } \quad Q(k)=\prod_{j=1}^{n}\left(q_{j}-k\right) .
$$

Thus (5.7) can be written as

$$
\Lambda_{n}-1=\Lambda_{n} \sum_{m=1}^{n} \frac{Q\left(p_{m}\right)}{\left(p_{m}-k\right) P^{\prime}\left(p_{m}\right)} .
$$

Dividing this equation by $\Lambda_{n}$ we see that this follows from the principal parts expansion for the meromorphic function $\Lambda^{-1}(k)=Q(k) /$ $P(k)$. This completes the proof of Theorem 5.1.

An independent proof, which does not make use of the specific representation of $F$ given by (2.6), will be given in [14].

\section{§6. Positivity of the $\tau$ Function; Special Solutions}

The coefficients of the operators $B_{n}$ are all obtained as derivatives of $\log \tau$; and so, in order for these functions to be real and 
regular, the $\tau$ function must be real and positive. In this section we establish the positivity of the $\tau$ function under certain restrictive conditions.

Recall ( $\S 1$ ) that the unstable case (KP I) corresponds to that where all the $x_{2 j}$ are imaginary, while KP II corresponds to that where all the $x_{j}$ are real. We now prove:

Theorem 6.1. Let $p(s)=-q(s)$ and let $\mu$ be a real positive measure in (2.6). Then $\tau$ is positive for $\mathrm{KPI}$.

Proof. For an $\mathrm{n} \times \mathrm{n}$ matrix $A$ it is a simple fact that $\operatorname{det}\|1+A\|$ is positive if all the eigenvalues $\lambda_{j}$ of $A$ are real and positive. This follows from the fact that

$$
\operatorname{det}\|1+A\|=\prod_{j=1}^{n}\left(1+\lambda_{j}\right) \text { 。 }
$$

This fact extends to the infinite dimensional case, so that to prove that $\tau$ is positive it suffices to prove that $F_{(x)}$ is positive definite, viz。 that $\left(F_{x} \Psi, \Psi\right) \geq 0$ for any $x_{1}$. We have

$$
\begin{aligned}
& \int_{x_{1}}^{\infty} \int_{x_{1}}^{\infty} F(x, z) \Psi\left(z_{1}\right) \Psi^{*}\left(x_{1}\right) d z_{1} d x_{1} \\
& =\int_{x_{1}}^{\infty} \int_{x_{1}}^{\infty} \int_{C} e^{\xi(x, p)-\xi\left(z_{1}-p^{*}\right)} d \mu(s) \Psi\left(z_{1}\right) \Psi^{*}\left(x_{1}\right) d z_{1} d x_{1} \\
& \int_{C} e^{\Phi_{2}(x)} \int_{x_{1}}^{\infty} e^{p x_{1}} \Psi^{*}\left(x_{1}\right) d x_{1} \int_{x_{1}}^{\infty} e^{p^{* z_{1}} z_{1}} \Psi\left(z_{1}\right) d z_{1} \\
& =\int_{C} e^{\Phi_{2}(x)}\left|\int_{x_{1}}^{\infty} e^{p x_{1}} \Psi^{*}\left(x_{1}\right) d x_{1}\right|^{2} d \mu(s)
\end{aligned}
$$

where

$$
\Phi_{2}(x)=\sum_{j=2}^{\infty} x_{j}\left(p^{j}-\left(-p^{*}\right)^{j}\right) .
$$

Since $\Phi_{2}(x)$ is real for KP I, the operator $F_{(x)}$ is positive definite under the conditions of the theorem.

Q.E.D.

The multi-soliton solutions to the KP hierarchy are easily constructed for KP I without any analytical difficulties, since the $\tau$ function is positive in that case (assuming that $R e p_{j}<0<R e q_{j}$ ). One can then study the analytic continuation of these solutions to $\mathrm{KP} \mathbb{I}$. Since the bilinear equations for the $\tau$ function are entirely analytic, 
there is no problem as long as $\tau$ does not vanish. As we shall see, however, the analytic continuation can introduce zeroes into $\tau$, and therefore poles into the solution. In addition, the analytic continuation to KP II is not necessarily real.

A one soliton solution is obtained from $F$ as given by (2.5) with $q=-p^{*}$. Define

$$
\begin{aligned}
\Phi(x ; p) & =\xi(x, p)-\xi\left(x,-p^{*}\right)=\sum_{j \text { odd }} 2 x_{j}\left(R e p^{j}\right)+i \sum_{j \text { even }} 2 x_{j}\left(\operatorname{Im} p^{j}\right) \\
& =R(x ; p)+i J(x ; p)
\end{aligned}
$$

and

$$
E(x ; p)=e^{\oplus(x ; p)} .
$$

In this notation the $\tau$ function is (cf. $\Delta(x)$ in $\S 2$ )

$$
\tau(x)=1+\frac{E(x ; p)}{2|\operatorname{Re} p|} \text {. }
$$

Now $\Phi(x ; p)$ is real for KP I, so $\tau$ is real and positive. By Theorem 3.1 the one-soliton solution of the KP hierarchy is

$$
u(x)=2\left(\frac{\partial}{\partial x_{1}}\right)^{2} \log \tau(x)=2|\operatorname{Re} p|^{2} \operatorname{sech}^{2}\{\Phi(x ; p)-\log 2|\operatorname{Re} p|\}
$$

For KP II, however, $\Phi(x ; p)$ takes complex values and $\tau$ has zeroes; the corresponding solution $u$ is therefore complex and has poles.

A real one-soliton solution to the KP hierarchy for KP II can be obtained by taking $p$ and $q$ real, with $p<0<q$ and $p+q \neq 0$. In that case we get

$$
\tau(x)=1+\frac{e^{\xi(x, p)-\xi(x, q)}}{q-p}
$$

which is real and positive for KP II.

The N-soliton solution of the KP hierarchy is obtained by taking $F$ as given in (2.7). The Fredholm series (3.1) terminates after $N$ terms. We can compute each of them explicitly by the methods of $\S 5$, thus giving the Hirota series for the $N$-soliton solution. The $n^{\text {th }}$ term in the series is

$$
\tau_{n}=\frac{1}{n !} \int \cdots \int E_{n} D_{n} d \mu
$$

where $E_{n}$ and $D_{n}$ are defined in $\S 5$ (cf. (5.4)). For the $N$-soliton case, the $\mu$ integrations are simply sums over $p_{1} \cdots p_{N}$. Hence, letting $P$ denote a subset of $\{1, \ldots, N\}$ we get 


$$
\tau_{n}=\frac{1}{n !} \sum_{j_{1} \cdots j_{N}} E(x ; P) D_{n}(P)=\sum_{|P|=n} E(x ; P) D_{n}(P)
$$

where

$$
E(x ; P)=\prod_{j \varepsilon P} a_{j} e^{\xi\left(x, p_{j}\right)-\xi\left(x, q_{j}\right)} \quad \text { and } D_{n}(P)=\operatorname{det}\left\|\frac{1}{q_{j}-p_{k}}\right\|_{j, k \varepsilon P_{\circ}}
$$

The second sum above is taken over all subsets $P$ of $\{1, \ldots, N\}$ of order $n$. The determinants $D_{n}(P)$ are easily evaluated by the PolyàSzegö identity. In the special case where $q_{j}=-p_{j}^{*}$ we get

$$
\frac{1}{2^{n}} \prod_{j \in P}\left|R e p_{j}\right|^{-1} \prod_{j<k \varepsilon P} \frac{\left|p_{k}+p_{j}\right|^{2}}{\left|p_{k}+p_{j}^{*}\right|^{2}}
$$

and the Hirota series (cf. [4]) is

$$
\tau(x)=\sum_{n=0}^{N} \frac{1}{2^{n}} \sum_{|P|=n} \prod_{j \in P} \frac{E\left(x ; p_{j}\right)}{\left|\operatorname{Re} p_{j}\right|} \prod_{j<k \in P} \frac{\left|p_{k}-p_{j}\right|^{2}}{\left|p_{k}+p_{j}^{*}\right|^{2}}
$$

where

$$
E\left(x, p_{j}\right)=a_{j} e^{\xi\left(x, p_{j}^{\prime}-\xi\left(x,-p_{j}^{*}\right)\right.}
$$

Note that $E_{n}(x ; P)$ and $D_{n}(P)$ are positive for $\mathrm{KP}$ I under our assumption that $q=-p^{*}$ in $(2.6)$. This observation can be extended to the general case and provides a second proof of Theorem 6.1.

We noted above that the one-soliton solution for KP II was complex for complex $p$ and had poles. However, there is a special "two soliton" solution which is real and regular for KP II. Namely, consider the soliton constructed by taking $\pm p$ and $\pm p^{*}$. The $\tau$ function for this configuration is easily calculated and found to be

$$
\tau(x)=1+\frac{e^{R(x ; p)} \cos J(x ; p)}{2|\operatorname{Re} p|}+\frac{e^{2 R(x ; p)}(\operatorname{Im} p)^{2}}{4|p|^{2}|\operatorname{Re} p|^{2}}
$$

It is easily seen that this function is positive for all $x$ if and only if

$$
\frac{\operatorname{Im} p}{|p|}>\frac{1}{2} \quad \text { hence } \quad \frac{\pi}{2}<\arg p<\frac{5 \pi}{6} \text {. }
$$

In analogy with the sine-Gordon solution, we may call this solution a "breather". Setting $x_{1}=x, x_{2}=y, x_{3}=t$, and all the other hierarchy variables equal to zero we have

$$
\begin{aligned}
& R(x ; p)=2 x \operatorname{Re} p+2 t \operatorname{Re} p^{3} \\
& J(x ; p)=2 y \operatorname{Im} p^{2} ;
\end{aligned}
$$

hence this solution is periodic in $y$ and decays in $x$. 
Another interesting configuration has been obtained by taking all the $p^{\prime} s$ and $q^{\prime} s$ on the imaginary axis (cf. [25]). Taking $p_{1}=i v_{1}$ and $q_{1}=i v_{2}, p_{2}=-i v_{2}, q_{2}=i v_{2}$, we find

$$
\tau(x)=1+\frac{2}{v_{2}-v_{1}}\left(\frac{E_{1}-E_{2}}{2 i}\right)+E_{1} E_{2} \frac{4 v_{1} v_{2}}{\left(v_{2}^{2}-v_{1}^{2}\right)}
$$

where

$$
E_{j}(x)=e^{\xi\left(x, p_{j}\right)-\xi\left(x, q_{j}\right)}
$$

for $j=1$, 2. Setting

$$
\begin{aligned}
& A(x)=\sum_{j=1}^{\infty} x_{2 j}(-1)^{j}\left(v_{1}^{2 j}-v_{2}^{2 j}\right)=x_{2}\left(v_{2}^{2}-v_{1}^{2}\right)+\cdots \\
& B(x)=\sum_{j=0}^{\infty} x_{2 j+1}(-1)^{j}\left(v_{1}^{2 j+1}-v_{2}^{2 j+1}\right)=x_{1}\left(v_{1}-v_{2}\right)+x_{3}\left(v_{2}^{3}-v_{1}^{3}\right)+\cdots
\end{aligned}
$$

we have $E_{1}(x)=e^{A(x)+i B(x)}, E_{2}(x)=e^{A(x)-i B(x)}$, and

$$
\tau(x)=1+\frac{2}{v_{2}-v_{1}} e^{A(x)} \sin B(x)+\frac{4 v_{1} v_{2}}{\left(v_{2}^{2}-v_{1}^{2}\right)^{2}} e^{2 A(x)} .
$$

In the case of KP II, all the variables are real, and it is easily verified that $\tau$ is positive for all real $x$ if

$$
\frac{1}{v_{1}}+\frac{1}{v_{2}}<4 \text {. }
$$

The solution is periodic in the odd variables and decays in the even variables as they tend to infinity. For KP I, when $x_{2 j+1}$ are real and $x_{2 j}$ are imaginary, the $\tau$ function is periodic in all variables, but complex.

The GLM equation makes no sense analytically when $p$ and $q$ are taken to lie on the imaginary axis, since then $F$ does not decay as $z \rightarrow \infty$. But one may construct the $\tau$ function for $\operatorname{Re} p<0<\operatorname{Re} q$, and then analytically continue it as $p$ and $q$ move onto the imaginary axis. The bilinear differential equations satisfied by $\tau$, being entirely algebraic in character, continue to hold as long as $\tau$ makes sense.

A $\tau$ function may be constructed, also as a determinant of a matrix of coefficients, to obtain the rational solutions; this construction will be given in [14].

\section{References}

[1] Ablowitz, M.J. and Fokas, A.S., On the inverse scattering of the time dependent Schrödinger equation and the associated Kadomtsev-Petviashvili (I) equation, Studies 
in Applied Math, 69 (1983), 211-228.

[2] Ablowitz, M. J., Bar Yaacov, D. and Fokas, A. S., On the inverse scattering transform for the Kadomtsev-Petviashvili Equation, Studies in Applied Math., 69 (1983), 135-143.

[3] Beals, R. and Coifman, R., The $D$-bar approach to inverse scattering and nonlinear evolutions, Physica 18D, (1986), 242-249.

[4] Date, E., Kashiwara, M., Jimbo, M. and Miwa, T., Transformation groups for soliton equations, in Proceedings of RIMS symposium on non-linear integrable systems, ed. Jimbo and Miwa, World Scientific Publishing co., Singapore, 1983.

[5] Gel'fand, I. M. and Dikii, L., Fractional powers of operators and Hamiltonian systems, Functional Anal. Appl., 10 (1976), 259-273.

[6] Hirota, R., Direct methods in soliton theory, in Solitons, Topics in Current Physics, 17 ; ed. R. K. Bullough and P. J. Caudrey, Springer-Verlag, Heidelberg, 1980.

[7] Jimbo, M., Miwa, T. and Ueno, K., Monodromy preserving deformation of linear ordinary differential equations with rational coefficients I, Physica 2D, (1981), 306-352.

[8] Manakov, S. V., The inverse scattering transform for time-dependent Schrödinger equation and Kadomtsev-Petviashvili equation, Physica 3D, (1981), 420-427.

[9] Novikov, S., Manakov, S. V., Pitaevskii, L. and Zakharov, V. E., Theory of Solitons, Plenum Publishing, New York, 1984.

[10] Oishi, S., Relationship between Hirota's method and the inverse spectral methodthe Korteweg-deVries equation's case-, Jour. Phys. Soc. Japan, 47 (1979), 1037-1038.

[11] Polyà, Szegö, Aufgaben und Lehrsätze aus der Analysis II, $3^{\text {rd }}$ ed. Springer-Verlag, 1974.

[12] Pöppe, C., Construction of solutions of the sine-Gordon equation by means of Fredholm determinants, Physica 9D (1983), 103.

[13] The Fredholm Determinant method for the KdV equations, Physica 13D, (1984), 137-160.

[14] General determinants and the $\tau$ function for the Kadomtsev-Petviashvili hierarchy, to appear Inverse Problem

[15] Rosales, R. R., Exact solutions of some nonlinear evolution equations, Studies in Applied Math. 59 (1978), 117-151.

[16] Riesz, F., Nagy, Sz., Functional Analysis, Frederick Ungar Publishing Co. New York, 1955.

[17] Ssto, M., Soliton equations as dynamical systems on infinite dimensional Grassmann manifolds, RIMS Kokyuroku, 439 (1981), 30.

[18] Segal, G. and Wilson, G., Loop groups and equations of KdV type, Publications I. H.E.S, 61 (1984), 5-65.

[19] Shabat, A. B., Concerning the Korteweg-deVries equation, Dokl. Akad. Nauk SSSR, 211 (1973), 1310-1313.

[20] Smithies, F., Integral Equations, Cambridge Univ. Press, Cambridge, 1965.

[21] Wilson, G., Commuting flows and conservation laws for Lax equations, Math. Proc. Camb. Phil Soc., 86 (1979), 131-143.

[22] - Infinite dimensional Lie groups and algebraic geometry in soliton theory, Phil. Trans R. Soc. London A, 315 (1985), 393-404.

[23] Zakharov, V.E. and Shabat, A. B., A scheme for integrating the nonlinear equations of mathematical physics by the method of the inverse scattering problem, Functional Analysis and its Applications, 8 (1974), 226-235.

[24] Zakharov, V. E., The inverse scattering method, in Solitons, ed. Bullough and Caudrey, Topics in Current Physics \#17, Springer, Heidelberg, 1980.

[25] Zakharov, V.E. and Manakov, S. V., Construction of higher dimensional nonlinear integrable systems and of their solutions, Functional Anal. Appl., 19 (1985), 89-100. 